\title{
$250 \mathrm{MHz} / \mathrm{GHz}$ Scintillation Parameters in the Equatorial, Polar, and Auroral Environments
}

\author{
SANTIMAY BASU, EILEEN MARTIN MACKENZIE, SUNANDA BASU, EMANOEL COSTA, \\ PAUL F. FOUGERE, SENIOR MEMBER, IEEE, HERBERT C. CARLSON, JR., \\ AND HERBERT E. WHITNEY
}

\begin{abstract}
Ionospheric scintillation effects encountered in the equatorial anomaly crest, polar cap, and auroral regions have been contrasted to provide information for the design and evaluation of the performance of multifrequency satellite communication links in these regions. The equatorial anomaly region is identified as the most disturbed irregularity environment where the amplitude and phase structures of $250 \mathrm{MHz}$ and $L$-band scintillations are primarily dictated by the strength of scattering rather than ionospheric motion. In the anomaly region, the spectra of intense amplitude scintillations at these frequencies are characterized by uniform power spectral density from the lowest frequency (10 MHz) to $4 \mathrm{~Hz}$ at $257 \mathrm{MHz}$ and to $1 \mathrm{~Hz}$ at $L$-band $(1541 \mathrm{MHz})$ and steep rolloff at higher fluctuation frequencies with power law indexes of -5 to -7 . Such structures are compatible with intensity decorrelation times of 0.1 and $0.3 \mathrm{~s}$ at 257 and $1541 \mathrm{MHz}$, respectively. The phase spectra at $244 \mathrm{MHz}$ are described by power law variation of psd with frequency with typical spectral indexes of -2.4. The strong scattering at VHF induces extreme phase rates of $200^{\circ}$ in $0.1 \mathrm{~s}$. The 90th percentile values of rms phase deviation at 244 MHz with $100 \mathrm{~s}$ detrend are found to be $16 \mathrm{rad}$ in the early evening hours, whereas amplitude scintillation can cover the entire dynamic range of $30 \mathrm{~dB}$ not only in the $250 \mathrm{MHz}$ band but at $L$-band as well. In the polar cap, the 50th and 90th percentile values of rms phase deviation at $250 \mathrm{MHz}$ for $82 \mathrm{~s}$ detrend are 3 and $12 \mathrm{rad}$, respectively, with comparable values being obtained in the auroral oval. The corresponding values for the $S_{4}$ index of scintillation are 0.5 and 0.8 in the polar cap, which are slightly higher than those recorded in the auroral oval. The power law index of phase scintillation at high latitudes is in the vicinity of -2.3 , which is not a result of very strong turbulence as in the equatorial region but is considered to be a consequence of shallow irregularity spectral indexes. The phase rates at auroral locations are an order of magnitude smaller than in the equatorial region and attain values of $100^{\circ}$ in $0.5 \mathrm{~s}$. The extreme variability of ionospheric motion in the auroral oval sensitively controls the structure of scintillations.

The long-term morphology (period 1979-1984) of intensity scintillations at $250 \mathrm{MHz}$ in the polar cap shows that, in addition to the absence of diurnal variation of scintillations, and the presence of an annual variation with a pronounced minimum during local summer, there exists a marked solar control of scintillation activity such that it abruptly decreases when the solar activity falls below a threshold level.
\end{abstract}

Manuscript received February 1, 1986; revised September 26, 1986. This work was supported in part by the Air Force Geophysics Laboratory under Contract F19628-84-K-0003 with Emmanuel College, and by the Defense Nuclear Agency.

S. Basu, P. F. Fougere, H. C. Carlson, Jr., and H. E. Whitney are with the Air Force Geophysics Laboratory, Hanscom Air Force Base, MA 01731 .

E. M. MacKenzie and S. Basu are with Emmanuel College, Boston, MA 02115

E. Costa was with Emmanuel College, Boston, MA. He is now with CE TUC-PUC/RJ Rua Marquês de São Vicente 225, 22453 Rio de Janeiro, Brazil.

IEEE Log Number 8612160.

\section{INTRODUCTION}

SATELlite communication links in the 250

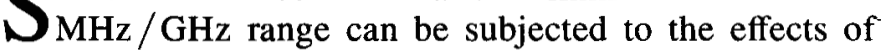
ionospheric scintillations which are caused by the irregularities of electron density in the $F$-region of the ionosphere. These irregularities impose random phase perturbations on the wavefront of a satellite signal during its passage through the ionosphere. As the wavefront with perturbed phase travels toward the ground, intensity and phase fluctuations develop across the wavefront due to phase mixing. In the case of an orbiting satellite, these intensity and phase fluctuations sweep past a receiver because of the satellite motion and the receiver output registers time variations of intensity and phase known as intensity or phase scintillations. On the other hand, in the case of a geostationary satellite, the motion of the ionospheric irregularities carries the intensity and phase variations across a fixed ray path and causes scintillations on a communication link. It should be remembered that the ray path from a satellite orbiting, at an altitude of 1000 $\mathrm{km}$, sweeps past the ionospheric irregularities assumed to be at $350 \mathrm{~km}$ at a speed of $\sim 3 \mathrm{~km} \mathrm{~s}^{-1}$. On the other hand, the speed of $F$-region irregularities relative to a receiver on the ground is typically on the order of $100 \mathrm{~m} \mathrm{~s}^{-1}$. As a result, the scintillation rate of orbiting satellite signals is generally an order of magnitude faster than in the case of geostationary satellites.

Scintillations cause both enhancements and fadings about the median level of the signal as the radio signals sweep across the irregular ionosphere. When these fadings exceed the specified fade margin of a link, its performance is degraded. The degree of degradation will depend on the magnitude of fadings relative to the margin, the duration of the fade, the rate of fading, the type of modulation, and the criteria of acceptability. On a global scale, the degradations are most serious for propagation paths which transit the low latitude irregularity belt around the magnetic equator and the high latitude environment encompassing the auroral oval and polar cap regions (cf. Fig. 1 given in [1]).

The morphology of scintillations has been studied for several years and documented for the equatorial, midlatitude, auroral, and polar cap regions. Intensity scintilla- 
tion measurements with orbiting and geostationary satellites provided the major database for such long-term studies [1]. The morphology of phase scintillations was developed by the use of multifrequency phase coherent DNA wide-band satellite transmissions in the equatorial [2] and auroral regions [3], [4]. More recently, near-stationary polar beacon satellites, which can be viewed at high elevation angles from high latitude stations, have been used to develop the morphology of phase and intensity scintillations in the auroral oval and polar cap regions [5]. In addition, case studies of ionospheric scintillations have been made in conjunction with rocket, radar, satellite in situ, and optical measurements [6]-[8]. These investigations have provided much insight into the mechanisms of irregularity formation and are expected to be helpful in developing predictive systems of scintillations based on geophysics [9].

In this paper, we first isolate very disturbed irregularity environments at both high and low latitudes, provide quantitative measures for the level of disturbance encountered by communication links in these regions, and analyze further the results in a form that can be used to evaluate communication system performance. We shall emphasize the difference between the structures of scintillation at high and low latitudes and isolate the appropriate parameters of concern to communication links operating in these two distinct disturbed regions of the globe.

\section{Data and Method of Analysis}

We have used both intensity and phase scintillation data from Thule, Greenland $\left(76.5^{\circ} \mathrm{N}, 68.7^{\circ} \mathrm{W}\right)$, a polar cap station; Goose Bay, Labrador $\left(53.3^{\circ} \mathrm{N}, 60.3^{\circ} \mathrm{W}\right)$, located in the nighttime auroral oval; and Ascension Island $\left(7.9^{\circ} \mathrm{S}, 14.4^{\circ} \mathrm{W}\right)$, an equatorial anomaly station where the critical frequency of the $F 2$ layer attains high values in the postsunset period when the ionospheric irregularities become most pronounced.

At Ascension Island, $244 \mathrm{MHz}$ signals from the geostationary satellite, Fleetsatcom, were recorded digitally by a computer-controlled phase-lock receiver. The processing of the phase and intensity scintillation data using the phase-lock system has been described earlier [10], [11]. This system was operated over a limited period of time, namely, Jan.-Feb. 1981 and Jan.-Feb. 1982, corresponding to periods of strong scintillation activity at this location. In addition to the above, total power receiving systems are employed to make routine recordings of signal intensity from Fleetsatcom at $244 \mathrm{MHz}$ and from the geostationary satellite, Marisat, at a variety of frequencies, namely, 257, 1541, and $3954 \mathrm{MHz}$. Round-the-clock observations by this total power system are recorded on chart recorders and manually analyzed to determine intensity scintillation magnitude over 15-min intervals [12]. During special campaign periods, however, the signals were recorded on magnetic tapes to perform spectral analysis and to evaluate both the first- and second-order parameters related to scintillation statistics.

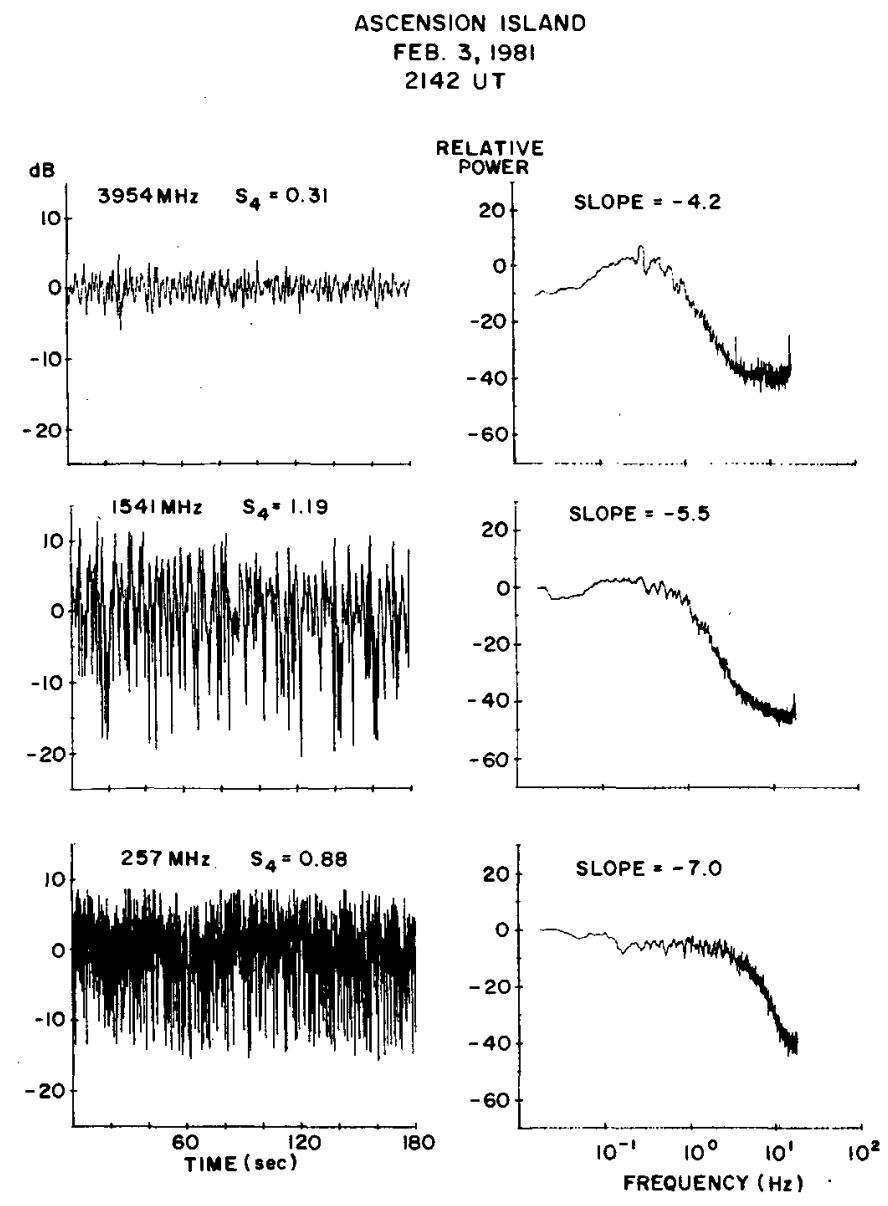

Fig. 1. A 3-min data segment of scintillations observed at 3954, 1541, and $257 \mathrm{MHz}$ at Ascension Island and their respective spectra.

At the high latitude stations, Thule and Goose Bay, 250 $\mathrm{MHz}$ signals from near-stationary polar beacon satellites were received at high elevation angles by both the computer-controlled phase-locked receiver and the total power system. In view of the periodic frequency updating of this satellite at 168-s intervals, the phase and intensity scintillation data could be processed over 82-s intervals spaced $168 \mathrm{~s}$ apart [5]. The total power system acquired intensity scintillation data on chart recorders on a round-the-clock mode which were analyzed manually as mentioned earlier.

\section{Results}

\section{A. Ascension Island}

During the last sunspot maximum period, the most severe scintillation activity was encountered at this equatorial station [13], [14]. Fig. 1 shows a sample of such an extreme case of scintillation activity which was recorded on multifrequency transmissions from Marisat. The lefthand panel shows, from the top, intensity scintillations at 3954,1541 , and $257 \mathrm{MHz}$ over a 3-min period in the early evening hours. The right-hand panel shows the corresponding power spectra for the samples with the power law index (slope) of the best fit straight line to the linear rolloff portions indicated on the diagram. On the left-hand panel, the second central moment of signal intensity, 
namely, the $S_{4}$ index of intensity scintillations defined in [15], is labeled to provide a quantitative measure of intensity fluctuations. At the lowest frequency, $257 \mathrm{MHz}$ shown in the bottom panel, the $S_{4}$ index of scintillations attains a value of 0.88 approaching the saturation condition of $S_{4} \sim 1$. The rate of fading is extremely fast and approaches the receiver response time of $0.1 \mathrm{~s}$. A receiver with larger dynamic range and faster response time would have recorded fading depths much larger than the $15 \mathrm{~dB}$ level registered in the figure. At higher frequencies, the phase perturbations are reduced so that scintillation magnitudes are expected to be less. In general, when scintillations are not very intense $\left(S_{4}<0.6\right)$, intensity scintillation magnitudes $\left(S_{4}\right)$ follow a frequency $(f)$ dependence of $f^{-1.5}$ shown in [16]. The middle panel, however, shows that at $1541 \mathrm{MHz}$ in the $L$-band, the $S_{4}$ index remains at the saturated level somewhat exceeding unity. This signifies that the irregularity environment is so intense that saturated scintillations are obtained at 1541 $\mathrm{MHz}$, and $257 \mathrm{MHz}$ scintillations are driven far into saturation. The level of activity can be gauged from the top panel which shows that even in the $C$-band (3954 MHz), scintillation with $S_{4}=0.31$ is obtained. From an engineering standpoint, this corresponds to a fluctuation of 6 $\mathrm{dB}$ [17].

The power spectra of scintillations obtained by the use of the fast Fourier transform (FFT) algorithnm are shown in the right-hand panel of Fig. 1 [18]. The spectrum of weak scintillations at $3954 \mathrm{MHz}$ is shown in the top panel. It is characterized by maximum power spectral density (psd) at a frequency $\left(f_{m}\right)$ of about $0.4 \mathrm{~Hz}$. This frequency can be expressed as $f_{m}=u / \sqrt{2 \lambda z}$ where $u$ is the drift speed of irregularities orthogonal to the propagation path, $\lambda$ is the radio wavelength, and $z$ is the slant range from the observing site to the irregularities [19]. For these observations, $\lambda=0.076 \mathrm{~m}, z=450 \mathrm{~km}$, and $f_{m}=0.4 \mathrm{~Hz}$, so that the value of $u$ is derived as $104 \mathrm{~m} \mathrm{~s}^{-1}$. This is in agreement with the observed values in the equatorial region where, during the early evening hours, the drift speed of $F$-layer irregularities with respect to the ground generally varies between $100-200 \mathrm{~m} \mathrm{~s}^{-1}$ [20]. In contrast to only a factor of 2 variation of drift speed in the equatorial region, the speed in the auroral oval can vary by a factor of 10 or more. This causes a considerable shift of $f_{m}$ even for a given geometry and a specified frequency as will be shown later in the paper. The other features to be noted are the slopes of the spectra on either side of the maximum psd. Since both psd and frequency are plotted on a logarithmic scale, a linear slope indicates a power law variation of psd with frequency. The spectrum of 3954 $\mathrm{MHz}$ scintillations indicates that at the low-frequency end, the variation can be approximated by $f^{+1}$ and the variation of psd at the high-frequency end can be expressed by $f^{-4.2}$. These spectral slopes are typically observed and have been related to the spectra of ionospheric irregularities [18] based on which the scintillation spectra have been successfully modeled [19].

The middle right-hand panel shows the spectrum of
$1541 \mathrm{MHz}$ signals which represents the case of saturated scintillations. In contrast to the $3954 \mathrm{MHz}$ spectrum discussed above, the spectral maximum in this case is broad and covers a frequency range of $0.1 \mathrm{~Hz}-1 \mathrm{~Hz}$. The highfrequency slope is also steeper indicating a power law index of -5.5 . The broad spectral width and steep spectral slopes are characteristics of strong intensity scintillations [21], [22], [19]. In fact, on the basis of the expression for $f_{m}$ given in the previous paragraph, which is valid for weak scintillations, one expects that at $1541 \mathrm{MHz}$, a lower value of $f_{m}$ will be obtained. Instead, $f_{m}$ extends to much higher values due to strong scattering [23]. Since the autocorrelation function and power spectra are Fourier transform pairs, the increased spectral width signifies a shorter autocorrelation interval. Henceforth, the time interval for 50 percent decorrelation will be referred to as the decorrelation time $(\tau)$. For practical purposes, it is found that the inverse of the frequency $f=2 \mathrm{~Hz}$, namely, $0.5 \mathrm{~s}$, where the psd falls to a level of $20 \mathrm{~dB}$ below the maximum, corresponds quite well to the decorrelation time.

The lowest panel in Fig. 1 shows the scintillation spectrum of $257 \mathrm{MHz}$ transmissions which is driven far into saturation. The spectral broadening is extreme in this case and extends to $4 \mathrm{~Hz}$. The spectral slope is steepest and corresponds to a power law index of -7 . The decorrelation time is found to be $0.13 \mathrm{~s}$ signifying an extreme fading rate. It should be noted that although the $S_{4}$ indexes for both 1541 and $257 \mathrm{MHz}$ are approximately unity indicating saturation, the decorrelation interval still varies, becoming shorter at the lower frequency which suffers stronger scattering.

Fig. 2(a) and (b) shows the cumulative distribution function (cdf) of signal amplitude for the $C$ - and $L$-band frequencies illustrated in Fig. 1. The cdf is a first-order statistic and is useful for defining the minimum margin requirements for communication links in nondiversity systems. The diagrams indicate the cdf for the observations (solid line) as well as the theoretical Nakagami $m$ distribution discussed in [24] which is indicated by dotted lines at the $m$ value ( $m=1 / S_{4}^{2}$ ) appropriate for the data sample. It can be seen that the theoretical distributions represent the observations quite well over a wide range of activity levels, being weak at $3954 \mathrm{MHz}$ and strong at $1541 \mathrm{MHz}$. For intense scintillations such as seen on 1541 $\mathrm{MHz}$, the cdf approaches a Rayleigh distribution ( $m=$ 1). The Nakagami $m$-distribution has been earlier shown to be useful for describing the effects of scintillations on satellite communication links [25].

In addition to the cdf which describes the probability distribution of the depth of fading, a statistical description of the fading rate is necessary to fully characterize the effects of scintillations on communication links. The information on the fading rate is contained in the power spectra of scintillations which we have described earlier. Another way of obtaining this information is to employ a level crossing technique. This gives the distribution of fade duration across a set of specified fading levels. Often, this representation is simpler and easier to interpret in re- 


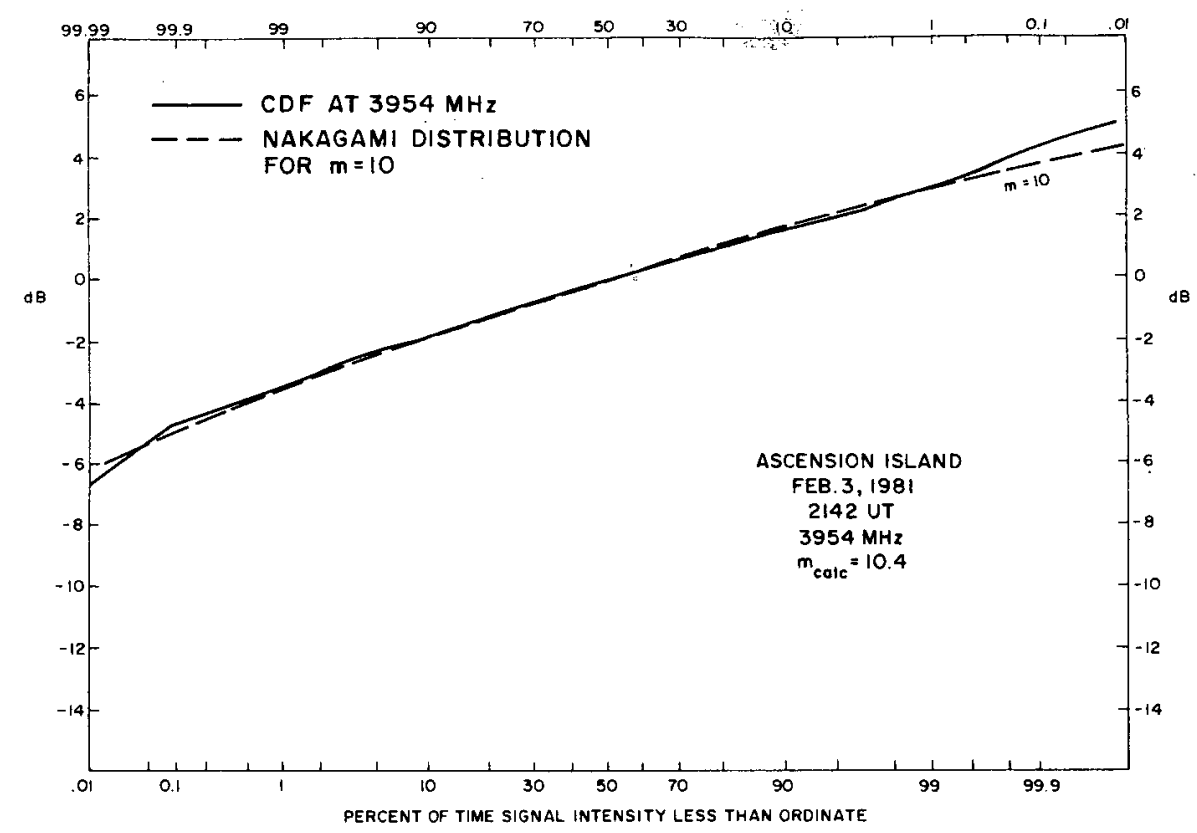

(a)

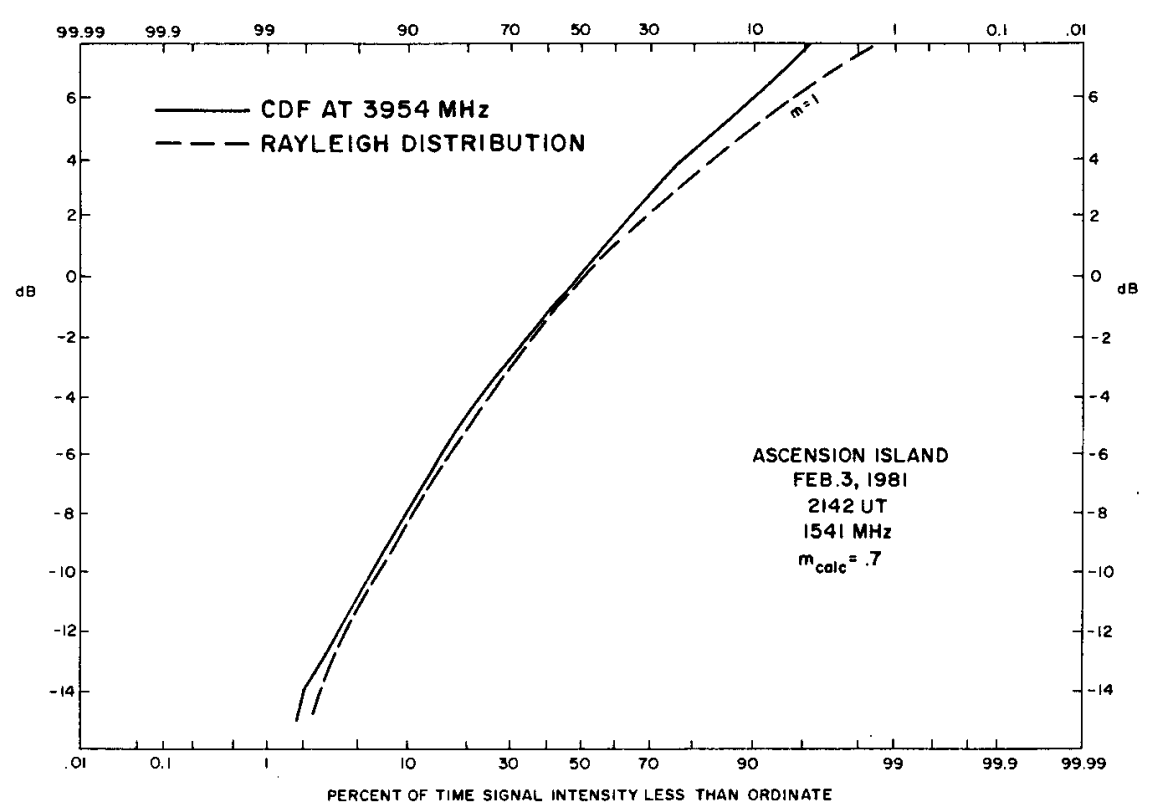

(b)

Fig. 2. (a) The cumulative amplitude distribution of scintillations at 3954 MHz shown in Fig. 1. (b) Same as in Fig. 2(a) for scintillations at $1541 \mathrm{MHz}$.

lation to systems applications. The left- and right-hand panels in Fig. 3 show the distribution of fades obtained at 3954 and $1541 \mathrm{MHz}$, respectively, over the first $1.5 \mathrm{~min}$ of the 3-min signal segments illustrated in Fig. 1. The shortest fade duration that could be measured in conformity with the data digitization rate was $0.04 \mathrm{~s}$. The lefthand panel shows the distribution of fade duration for the $3954 \mathrm{MHz}$ scintillation sample. Four separate fade depth levels at $1 \mathrm{~dB}$ intervals are found to be appropriate for this sample of weak scintillation. At the $-1 \mathrm{~dB}$ fade level, the flat top portion of the curve commences to slope downwards at a fade duration of $0.2 \mathrm{~s}$, which represents the longest fade duration at this fade level. The maximum number of fades encountered at the $-1 \mathrm{~dB}$ level is 54 . This number reduces with decrease in fade duration and attains a count of 27 , i.e., 50 percent of the maximum count at a fade duration of $0.08 \mathrm{~s}$. At the next lower fade level of $-2 \mathrm{~dB}$, the maximum number of fades reduces to 22 . The $-4 \mathrm{~dB}$ fade level lies on the abscissa indicating that fades do not reach this level. The right-hand panel shows the corresponding distribution for $1541 \mathrm{MHz}$ scintillation which is much more intense and is, in fact, sat- 

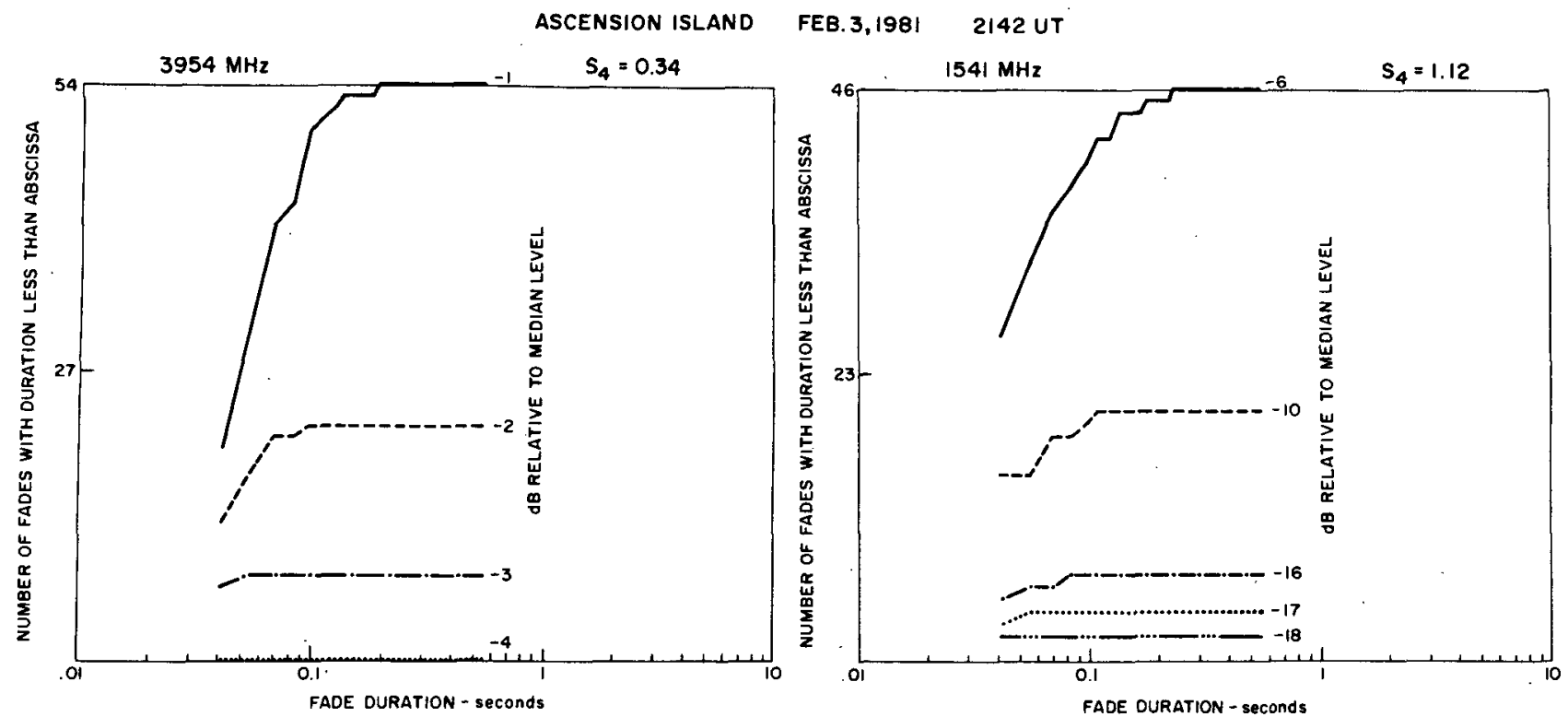

Fig. 3. Distribution of the fade durations for the period of weak scintillations at $3954 \mathrm{MHz}$ and strong scintillations at $1541 \mathrm{MHz}$ illustrated in

Fig. 1:

urated at $S_{4}=1.12$. In this case, the fade levels are chosen at wider intervals. The distribution is interesting in the sense that the number of fades is drastically reduced at higher fade levels exceeding $-16 \mathrm{~dB}$. Thus, although the fade depth of strong $1541 \mathrm{MHz}$ scintillations exceeds $20 \mathrm{~dB}$, the number of fades at $-16 \mathrm{~dB}$ level in one 1.5 min interval is only 7 and the maximum fade duration does not exceed $0.1 \mathrm{~s}$.

We shall next concentrate on the results of both intensity and phase measurements at Ascension Island performed with the computer-controlled receiver discussed in Section II. These measurements were performed by the use of $244 \mathrm{MHz}$ transmissions from Fleetsatcom. The intensity and phase scintillation data were analyzed over successive $150 \mathrm{~s}$ periods. Since the $257 \mathrm{MHz}$ intensity scintillation data acquired from the Marisat satellite have been discussed in earlier publications [14], [19], we shall comment primarily on the phase scintillation data which are quite unique and are being reported here for the first time.

Fig. 4 shows the 50th and 90th percentiles of the $S_{4}$ index of intensity scintillation (defined earlier) and rms phase deviation $\left(\sigma_{\phi}\right)$ over 150 -s periods (detrended with a $0.01 \mathrm{~Hz}$ filter) as a function of local time. The time interval corresponds to the postsunset to postmidnight period (20-04 LT) (local time) when equatorial $F$-region irregularities are most intense and abundant. The period Jan.-Feb. 1981 corresponds to a period of high scintillation occurrence as previously mentioned. The number of data points in each 2 -h block is indicated along the abscissa. It may be noted that in the early evening hours between 20-22 LT, both the median and 90th percentile values of $S_{4}$ and $\sigma_{\phi}$ are highly elevated. The intensity scintillation data saturated at a level of approximately $S_{4}=$ 0.8 because of receiver constraints. With the Marisat sat-

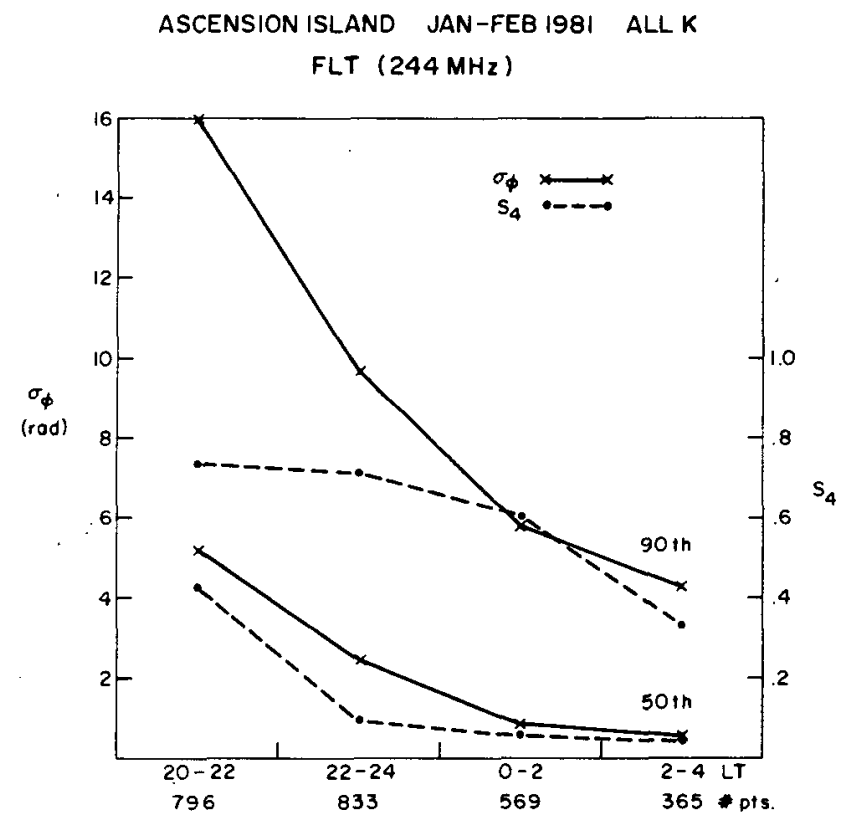

Fig. 4. The median (50th percentile) and 90th percentile values of phase and intensity scintillations at $244 \mathrm{MHz}$ in terms of four 2-h blocks of local time (LT) during Jan.-Feb. 1981 at Ascension Island.

ellite, we have shown that both the $257 \mathrm{MHz}$ and $L$-band intensity scintillations at this station become saturated with $S_{4} \approx 1.0$. The saturation of intensity scintillation data at about 0.8 did not, however, affect phase measurements. It may be noted that the 90th percentile of $\mathrm{rms}$ phase deviation in the 20-22-h block is as large as $16 \mathrm{rad}$ with $100 \mathrm{~s}$ detrend. In a similar propagation geometry, the values of rms phase deviation may be scaled to shorter detrend intervals $\left(T_{d}\right)$ by multiplying the given values by $\left[T_{d} / 100\right]^{(p-1) / 2}$, where $p(\sim 2.5)$ is the phase spectral index [26]. From Fig. 4, it may also be noted that the 


\section{ASCENSION ISLAND}

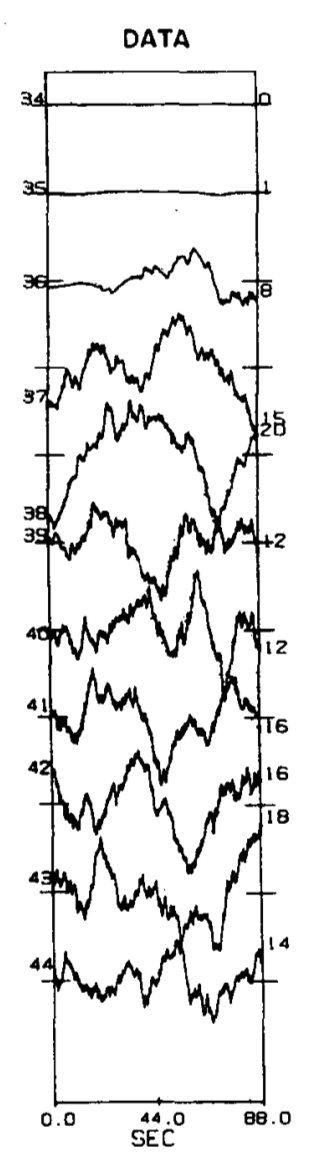

SPECTRA (MEM 3O)

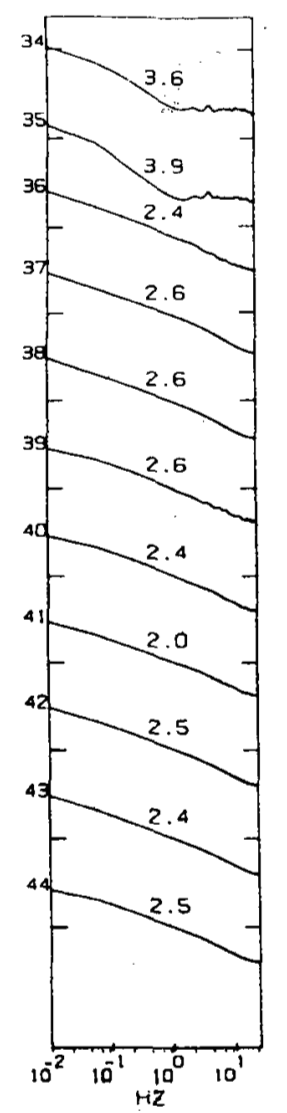

FË: 3,1981

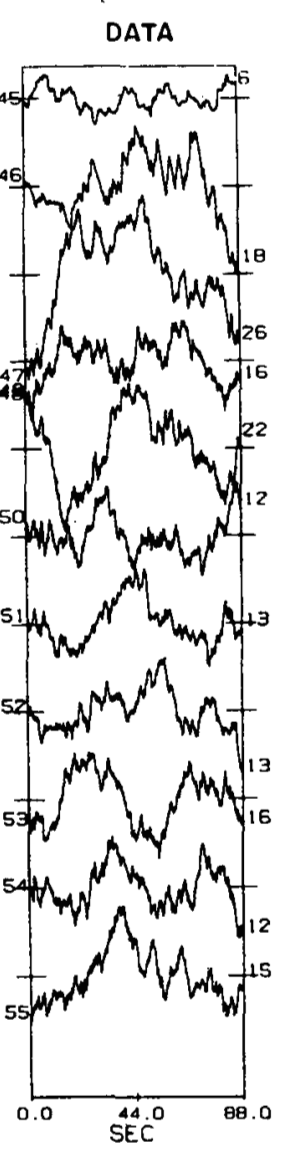

222430 UT

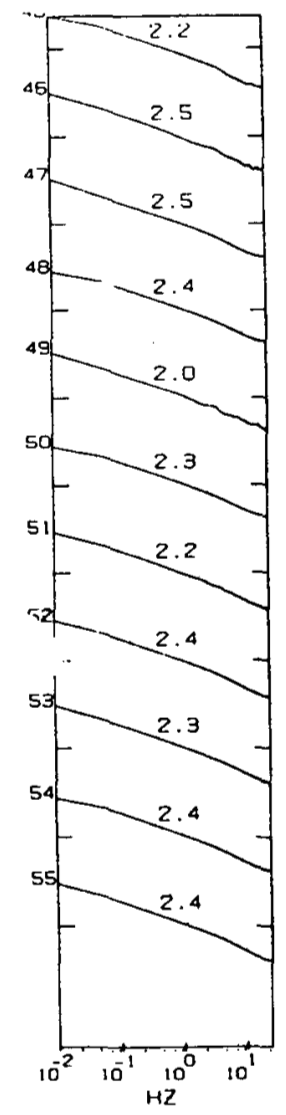

Fig. 5. Phase scintillation data segments at $244 \mathrm{MHz}$ over 88 -s intervals and their spectra in alternate panels acquired at Ascension Island on Feb. 3,1981 .

50th and 90th percentile values of these scintillation parameters decrease sharply with increasing local time owing to the decay of irregularity strength.

We shall now discuss the $244 \mathrm{MHz}$ phase scintillation data that were acquired from the Fleetsat satellite at Ascension Island on Feb. 3, 1981. Multifrequency intensity scintillation data from the Marisat satellite for this day have already been presented earlier. Fig. 5 shows a sequence of phase scintillation data over successive 88-s intervals and their spectra obtained by the maximum entropy method [27] in the adjacent panels. The signal segments and their spectra are numbered on the left-hand side of each panel for ease of comparison. The rms phase deviation in radians for each 88-s interval is indicated on the right-hand side of the data segments. The intervals between the tic marks on the data panel represent $50 \mathrm{rad}$ and on the spectral panels psd of $80 \mathrm{~dB}$. The initial data segments with no activity are not shown and the panels start from the 34th data segment, which commences at 222430 universal time (UT). The first two data segments (34 and 35) show very little phase variation. The corresponding phase spectra show that the noise floor exterids from the Nyquist frequency of $25 \mathrm{~Hz}$ to about $0.2 \mathrm{~Hz}$. Phase power spectral densities (psd) are noticeable only at lower frequencies. A sudden onset of scintillations may be noted in the third panel. The corresponding spectra indicates an abrupt increase of psd at all frequencies up to $25 \mathrm{~Hz}$. The numbers alongside the spectra indicate the magnitude of maximum instantaneous spectral slopes. The spectral slopes attain values ranging between -2 to -2.5 during the period of scintillations, but remain much higher before the onset of scintillations as may be noted from the first two spectra. It is worthwhile to recall that the spectral slope of intensity scintillations at a similar frequency (cf. Fig. 1) and a similar period of intense activity is very steep. This is caused by the refractive effects of large scale irregularities of electron density on the structure of intensity scintillations [21], [22]. Until recently, one expected that the spectra of weak intensity scintillations and phase scintillations of any magnitude would reflect the slope of irregularity spectra in the ionosphere. In fact, the slope of -4.2 in the weak scintillation spectrum at $3954 \mathrm{MHz}$ as shown in Fig. 1 reflects very well the average one-dimensional irregularity spectral slope of -3.5 at small scales [18]. The spectral slopes of strong phase scintillations at $244 \mathrm{MHz}$, however, indicate shallow spectral slopes in the vicinity of -2.5 . The apparent discrepancy has been resolved by Rino and Owen [28]. They simulated an 


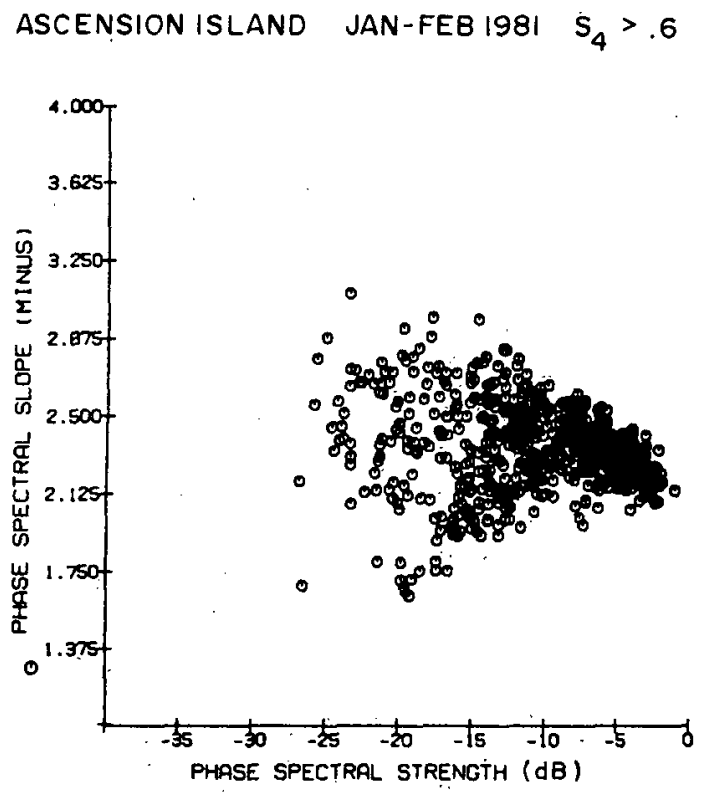

Fig. 6. A scatter plot of the phase spectral slope against phase spectral strength (dB) at Ascension Island at $244 \mathrm{MHz}$. The slope approaches a value of -2 at high phase spectral strengths.

ionospheric phase screen, allowed a radio wave to propagate through it, and computed numerically the phase structure and phase spectra at various distances from the screen. They showed that due to the diffractive effects of phase in a strong scattering medium, the phase structure develops large, near-discontinuous phase changes (termed "cycle slips") and yields phase spectra with slopes approaching -2 .

Fig. 6 shows a scatter plot of phase spectral slope versus phase spectral strength in decibels which is defined as the phase psd at $1 \mathrm{~Hz}$ when the $S_{4}$ index exceeds 0.6 , i.e., for strong scintillations. The data were acquired at Ascension Island during Jan.-Feb. 1981. At low values of phase spectral strengths or high decibel numbers, phase spectral slopes show a wide scatter between -2 and -2.9 in Fig. 6; but at high values of phase psd, the slopes approach a value of -2 . This provides observational support to the "cycle slip" argument presented in the previous paragraph.

In Fig. 7, we show an interesting plot of intensity decorrelation time at $244 \mathrm{MHz}$ on a logarithmic scale against phase spectral strength (decibels) for strong scintillations $\left(S_{4}>0.6\right)$. It may be noted that with increased phase spectral strengths in this regime, the intensity scintillations systematically develop finer structures on the ground so that the decorrelation time decreases from about 1 to $0.05 \mathrm{~s}$. By using the method outlined in [26], it is possible to derive the average spectral slope from the slope of the best fit straight line to the scatter plot of Fig. 7. The scatter diagram indicates a spectral slope of -2.4 . This is in very good agreement with the phase spectral slopes of the data segments illustrated in Fig. 5.

The results shown in Figs. 6 and 7 imply that in the disturbed equatorial ionosphere, the structures of inten-
ASCENSION ISLAND JAN-FEB $1981 \quad . \mathrm{S}_{4}>.6$

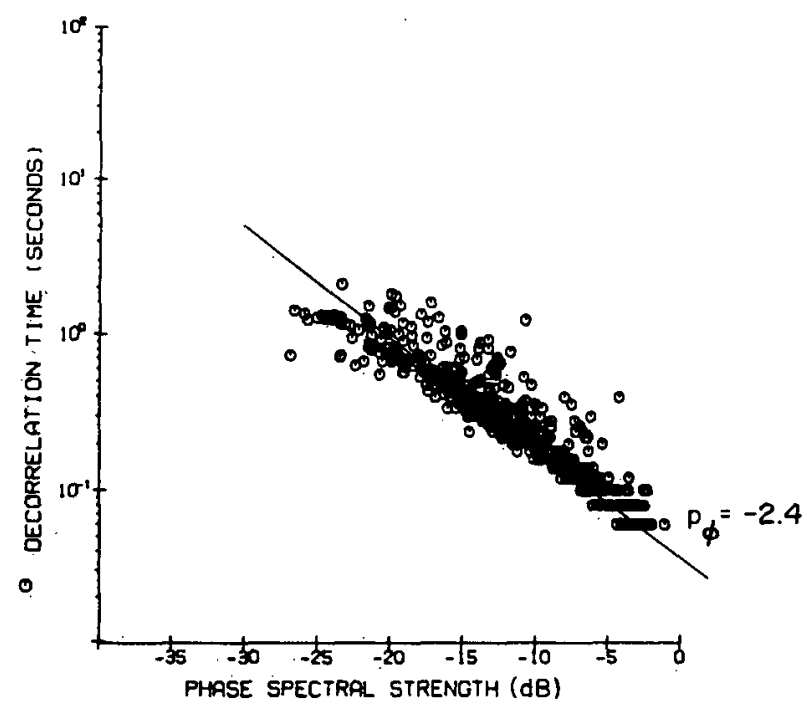

Fig. 7. A scatter plot of intensity decorrelation time against the phase spectral strength of $244 \mathrm{MHz}$ scintillations recorded at Ascension Island. The theoretical dependence of the two parameters in the strong scatter regime for a phase spectral index $p_{\phi}=-2.4$ is indicated by the straight line.

sity and phase scintillations are controlled by the strength of scattering. The decorrelation time is a function of the spatial structure of intensity variation on the ground as well as the ionospheric drift which controls the motion of the spatial structure past a receiving antenna. The fact that the decorrelation time is so well ordered in terms of the phase spectral strength indicates that the drift velocity does not vary much between the postsunset and the midnight period, and it is the strength of the irregularities which control primarily the fading pattern of scintillations. This is, however, not true at high latitudes where the phase spectral strength of the integrated strength of irregularities is not as intense as in the equatorial region, but the drift velocity can vary by an order of magnitude within a short interval of time. At high latitudes, therefore, the fading pattern is primarily controlled by the irregularity drift.

From Fig. 5 it was evident that in intense equatorial phase scintillation events, considerable power resides in the regime of high fluctuation frequencies. This is equivalent to stating that the probability of having a high rate of change of phase is considerable. The phase rate, on the other hand, is a measure of the Doppler spread the signal undergoes in traversing the irregularities of the ionosphere. In Fig. 8, the probability distribution of the phase rate in phase scintillations has been computed over $0.1-\mathrm{s}$ intervals during a $30-\mathrm{min}$ period of intense scintillations at Ascension Island. Since the Nyquist frequency is 25 $\mathrm{Hz}$, the phase rate could have been computed over a much shorter time interval. However, at small sampling intervals, the noise becomes comparable to the signal and the derived phase rates would have been contaminated by the phase rate of noise signals. In Fig. 8(a), the occurrence 


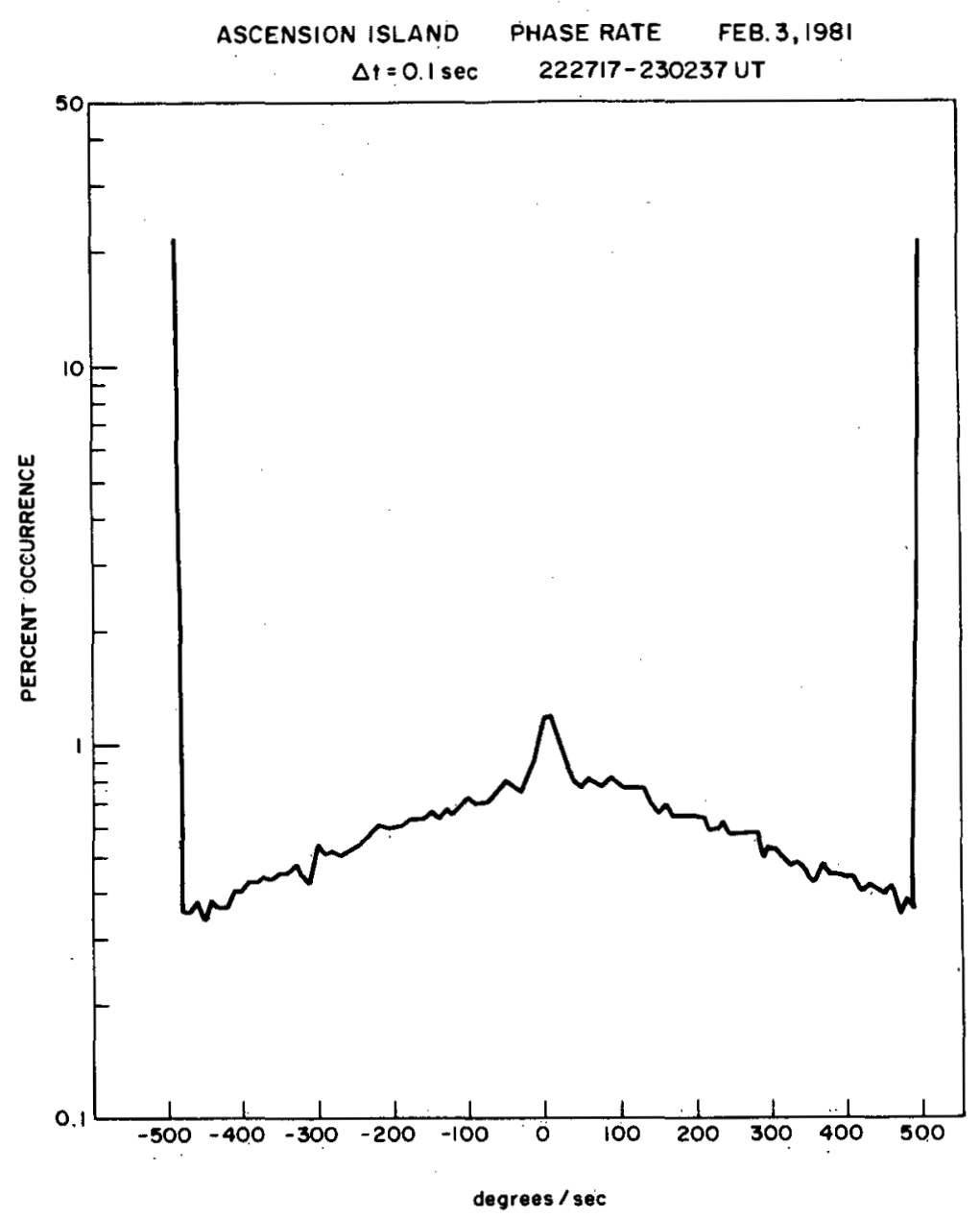

(a)

Fig. 8. (a) The distribution of phase rate of $244 \mathrm{MHz}$ scintillations at Ascension Island. The high values at the two ends indicate percent occurrence of values $>500^{\circ} \mathrm{s}^{-1}$.

of phase rate between $\pm 500^{\circ} \mathrm{s}^{-1}$ has been plotted. It is found that nearly 20 percent of the total population $\left(10^{5}\right.$ points) contains phase rates in excess of $\pm 500^{\circ} \mathrm{s}^{-1}$. The distribution of population with extreme phase rates has been plotted in Fig. 8(b) over a wider range extending from \pm 500 to $\pm 2300^{\circ} \mathrm{s}^{-1}$. It may be noted that phase rates as high as $2000^{\circ} \mathrm{s}^{-1}$ or $2^{\circ} \mathrm{ms}^{-1}$ occur in 0.1 percent of the population with extreme phase rates which, in turn, represents 20 percent of the total number of observations during approximately a half-hour period. Such extreme phase rates have considerable deleterious effects on radar systems. Since the phase scintillation magnitude varies inversely as frequency [29], the above phase rate may be scaled from the $244 \mathrm{MHz}$ observations to $2.4 \mathrm{GHz}$ as $0.2^{\circ}$ $\mathrm{ms}^{-1}$.

\section{B. Thule, Greenland}

At Thule, a polar cap station, the phase and intensity scintillation data were acquired by receiving $250 \mathrm{MHz}$ transmissions from the near-stationary Air Force satellites at high elevation angles. The ionospheric intersection of the ray path covered a corrected geomagnetic latitude range of $85-89^{\circ} \mathrm{N}$. Since scintillations are better ordered in terms of corrected geomagnetic latitude (CGLAT) and magnetic local time (MLT) at high latitudes, we shall use this system of coordinates as described in [30]. The phase and intensity measurements were made with the computer-controlled phase-lock receiver during specified intervals as mentioned in Section II.

The morphology of intensity scintillations [31] and that of both phase and intensity scintillations [5] from Thule have been discussed earlier. In this paper, we shall present phase and intensity scintillation data during Jan.-Feb. 1982 not previously published, and use it to contrast its behavior against the Ascension Island observations. We have also extended the intensity scintillation morphology further through the years of low sunspot number to illustrate the control of solar activity on polar cap scintillations.

Fig. 9 shows the variation of $250 \mathrm{MHz}$ intensity scintillation index $\left(S_{4}\right)$ and rms phase deviation $\left(\dot{\sigma}_{\phi}\right)$ over 82 $s$ intervals with magnetic local time as observed at Thule 


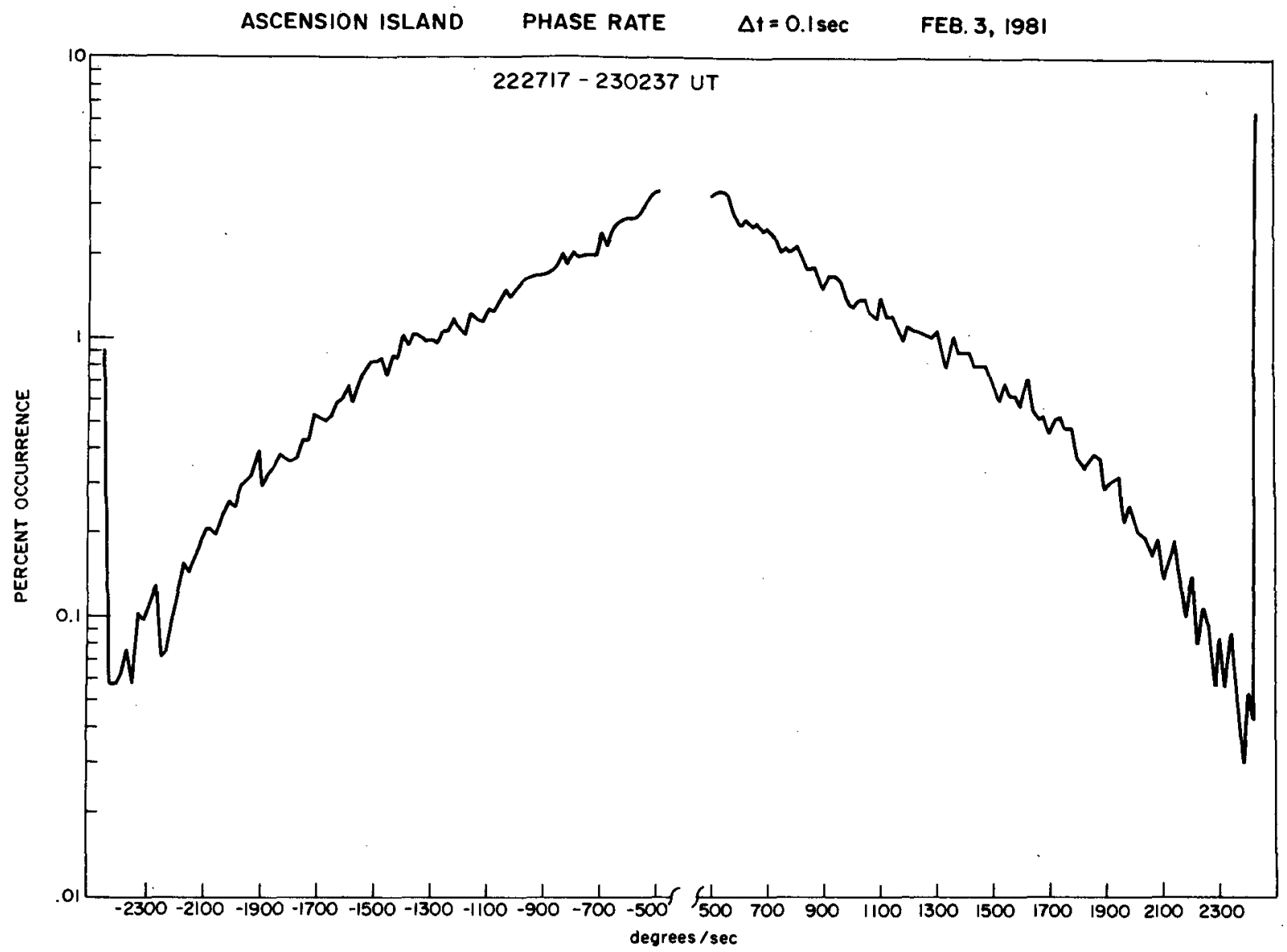

(b)

Fig. 8. (Continued) (b) The distribution of the population with extreme phase rates which corresponded to the two ends of the diagram in Fig. 8(a).

THULE JAN-FEB 1982 ALL K POLAR BEACON $244 \mathrm{MHz}$

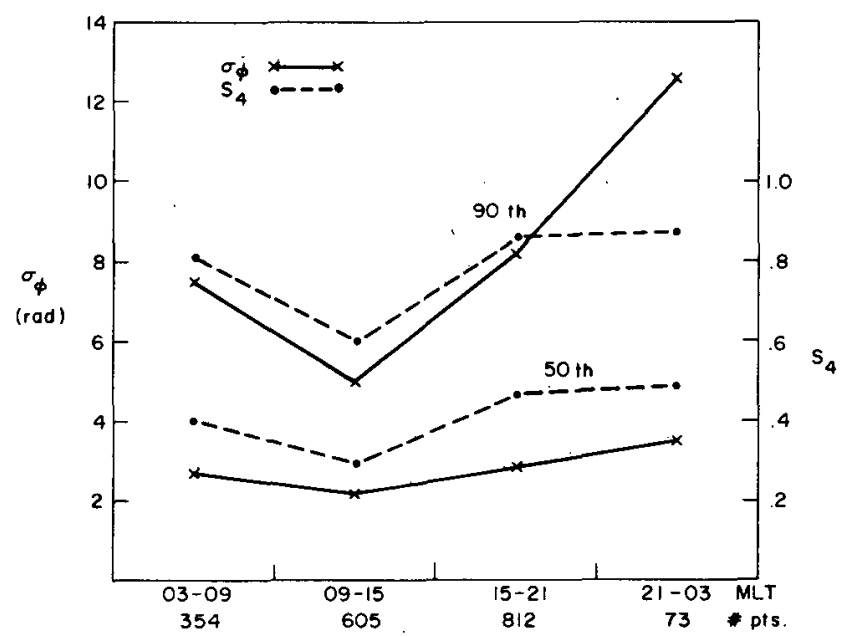

Fig. 9. The median (50th percentile) and the 90th percentile values of phase and intensity scintillation at $250 \mathrm{MHz}$ in terms of four 6-h MLT blocks observed at Thule.

during Jan.-Feb. 1982. It may be noted that at Thule, the scintillation activity during years of high sunspot numbers persists at all hours, the median value of phase ranging between 3 and 5 rad over the entire diurnal period. This behavior is in sharp contrast to that at Ascension Island, an equatorial station, where strong scintillations are, in general, confined between the postsunset to midnight hours. In view of this, the scintillation statistics in Fig. 4 were derived between 20-04 LT. At auroral locations, such as Goose Bay, scintillations on magnetically quiet days are again confined to nighttime periods as discussed in [5]. Thus, an absence of diurnal variation of scintillations during periods of high solar activity is a feature confined only to the polar cap. The level of $250 \mathrm{MHz}$ scintillations is also high, the 90th percentiles of rms phase attaining values as high as 12 rad and intensity scintillations remaining near saturation with $S_{4} \sim 0.9$.

The long-term statistics of $250 \mathrm{MHz}$ intensity scintillations derived with total power receiving systems are shown in Fig. 10. The figure shows the variation of scintillation occurrence at various fade depths for 1979-1984. The highest fade level of $\geq 20 \mathrm{~dB}$ represents a peak-topeak fluctuation level of $\geq 28 \mathrm{~dB}$. The scintillations minimize during the summer months so that the annual variation becomes most conspicuous. During the sunlit period, the high conductivity at $E$-region heights $(\sim 100 \mathrm{~km})$ is not favorable for the maintenance of irregularities in the overlying $F$-region of the ionosphere [32]. In other periods, particularly during the autumnal equinox, fade depths as large as $20 \mathrm{~dB}$ with 20 percent occurrence could 


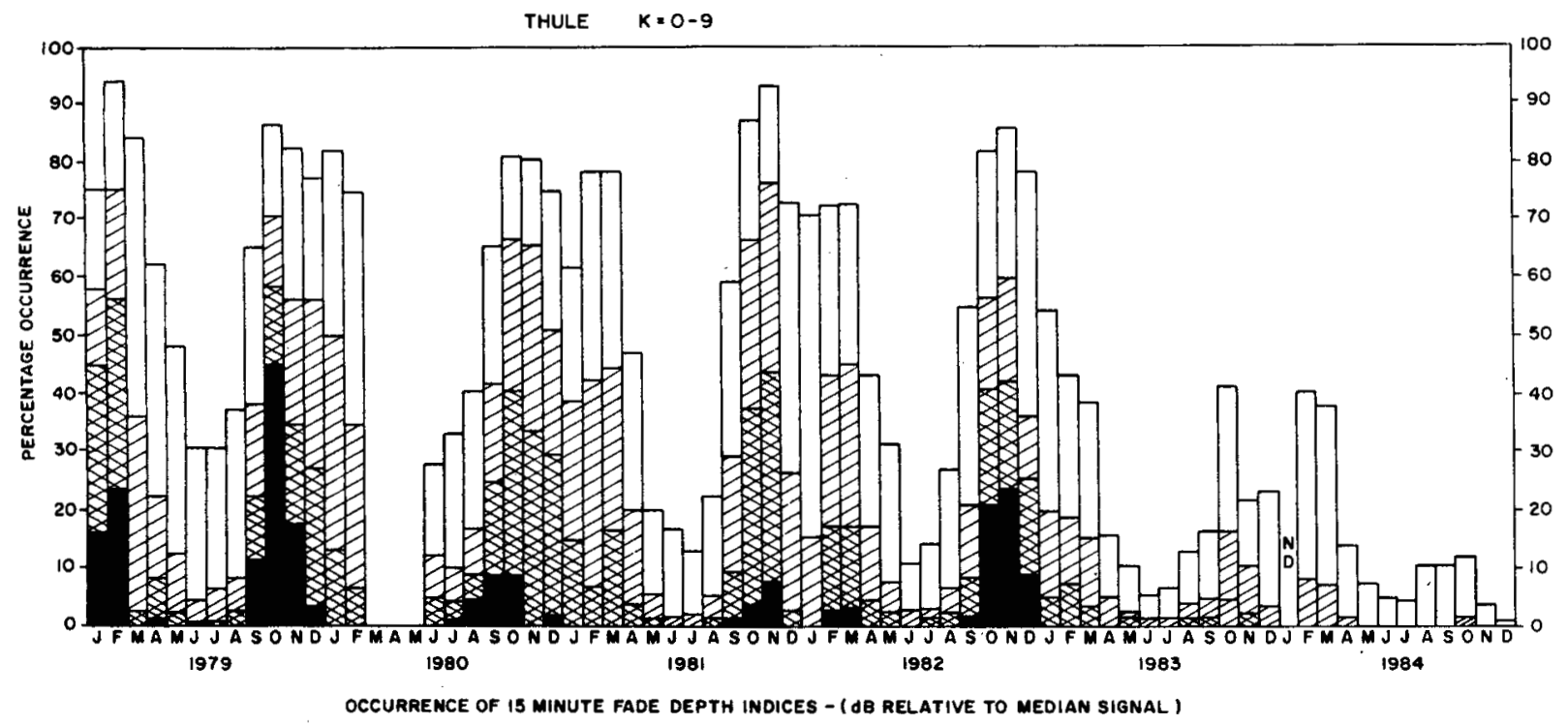

Fig. 10. Thule intensity scintillation statistics at $250 \mathrm{MHz}$ during 1979-1984.

be obtained up to 1982 . The scintillation activity did not vary much during the period 1979-1982 when sunspot numbers varied between 200 and 100 . With further reduction of sunspot numbers, scintillations showed an abrupt decline during 1983 and 1984 . Thus, there seems to be a lower threshold of solar activity below which irregularities of sufficient integrated strength are not encountered.

Fig. 11 shows the occurrence contours of fade levels at $250 \mathrm{MHz}$ that exceed $10 \mathrm{~dB}$ on both a monthly and diurnal basis. As pointed out in the previous paragraphs, the annual variation of scintillations is the more prominent feature and the diurnal variation is virtually absent at Thule. However, there emerges a diurnal pattern in the Oct.Nov. period which becomes most conspicuous during the low sunspot years of 1983 and 1984. The maximum activity during these months seems to be confined to the afternoon period around 15 MLT.

The strong scintillation events with $S_{4}$ exceeding 0.6 , that could be recorded by the phase-lock receiving system at $250 \mathrm{MHz}$, were sorted. The intensity decorrelation time and phase spectral strengths were determined and their mutual dependence is plotted in Fig. 12. The best fit straight line through the scatter plots corresponded to the theoretical dependence [26] expected for the case of strong scattering with phase spectral slope of -2.2 . It signifies that during strong scintillations at Thule, the intensity scintillation structure is largely governed by the phase spectal strength. Increased scatter around the best fit line signifies that the drift speed may vary by a factor of 3-4.
On the other hand, the scatter in Fig. 7 is much less, indicating that the variation of drift speed is small in the equatorial region. At the auroral station of Goose Bay, a similar plot (not shown here) indicates considerable scatter as well due to the high variability of drift speeds.

\section{Goose Bay, Labrador}

We shall not discuss in great detail the features of scintillations at Goose Bay, an auroral station, but refer to [5] which compared the characteristics of polar cap and auroral scintillations. In short, the auroral station shows a well-ordered diurnal variation of scintillations with a nighttime maximum and daytime minimum, whereas we have shown the absence of such ordering in the polar cap region, such as Thule. The auroral scintillation magnitudes are, on the average, similar to that in the polar cap although, on occasions, more active conditions prevail in the polar cap. The polar cap scintillation pattern exhibits the presence of discrete structures, whereas in the auroral oval, intense scintillation events continue for hours without showing much variation in magnitude. The major feature of an auroral station is the extreme variability of drift speed. It is not uncommon to observe a change in speed from $100 \mathrm{~m} \mathrm{~s}^{-1}$ to $1000 \mathrm{~m} \mathrm{~s}^{-1}$ within a 30 -min period. This variability in speed affects greatly the temporal structure parameters of scintillations such as the decorrelation time or the rate of change of phase and intensity. Fig. 13 shows the rate of change of intensity at $250 \mathrm{MHz}$ encountered on two successive nights, Mar. 7 and 8, 1982. The scheme of the plot is similar to the phase rate plot in Fig. 


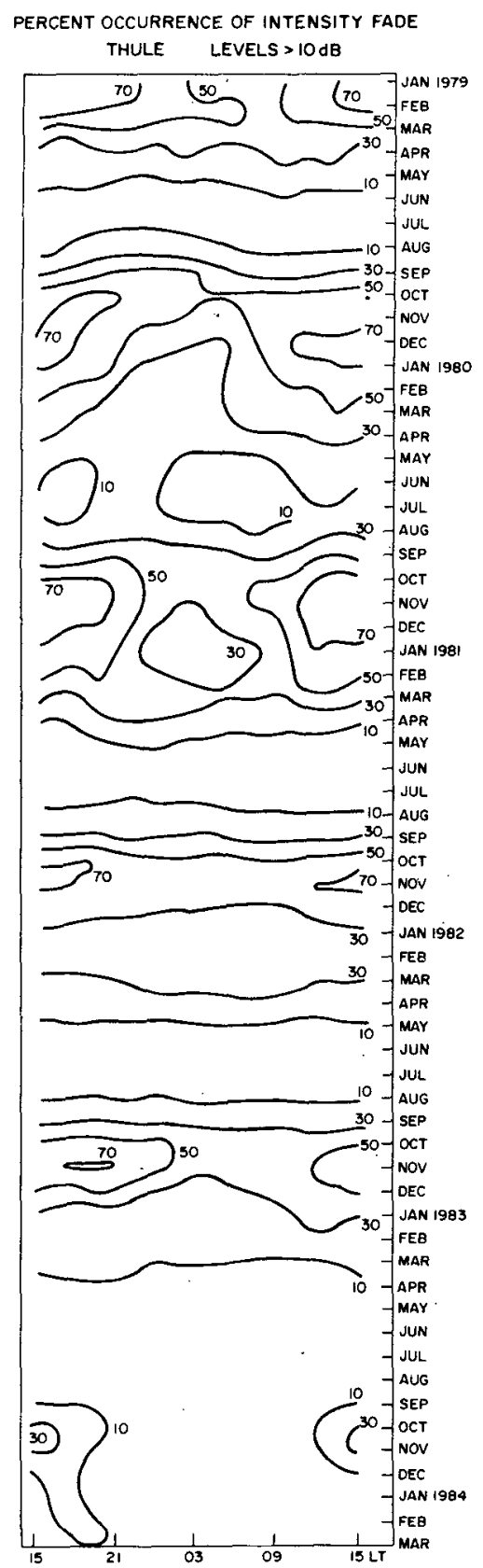

Fig. 11. Intensity scintillations at Thule at $250 \mathrm{MHz}$ on a seasonal and local time basis. The contour levels represent percent occurrence.

8(a) for A'scension-Island, except for the longer data interval $(0.5 \mathrm{~s})$ used to compute the rates at the auroral location. Shorter time intervals could not be used here because of possible noise contamination. At the equatorial station, intervals as short as $0.1 \mathrm{~s}$ could be used to compute phase rates as high levels of ionospheric turbulence developed high-frequency structures much above the noise floor. Fig. 13 shows that at Goose Bay, on Mar. 7, 1982, the intensity rate of $3 \mathrm{~dB} \mathrm{~s}^{-1}$ occurs in 0.1 percent of the total population, whereas it increases to about $7 \mathrm{~dB} \mathrm{~s}^{-1}$ at the same 0.1 percent level on Mar. 8, 1982. Intensity spectral studies revealed that the irregularity drift speed was $65 \mathrm{~m} \mathrm{~s}^{-1}$ on Mar. 7, 1982, and increased to $390 \mathrm{~m}$ $\mathrm{s}^{-1}$ on Mar. 8, 1982 [5]. The sixfold increase in drift
THULE JAN-FEB $1982 \quad \mathrm{~S}_{4}>.6$

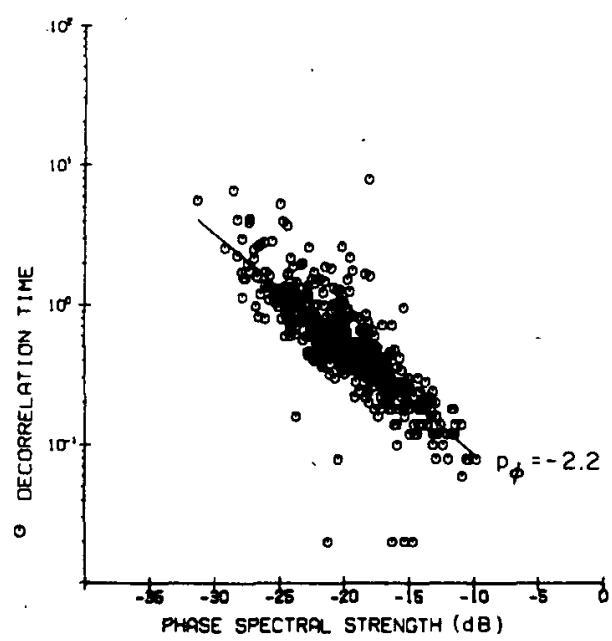

Fig. 12. A scatter plot of the intensity decorrelation time against phase spectral strength observed at Thule at $250 \mathrm{MHz}$. The theoretical dependence of the two parameters for a phase spectral index of -2.2 is indicated by the straight line.

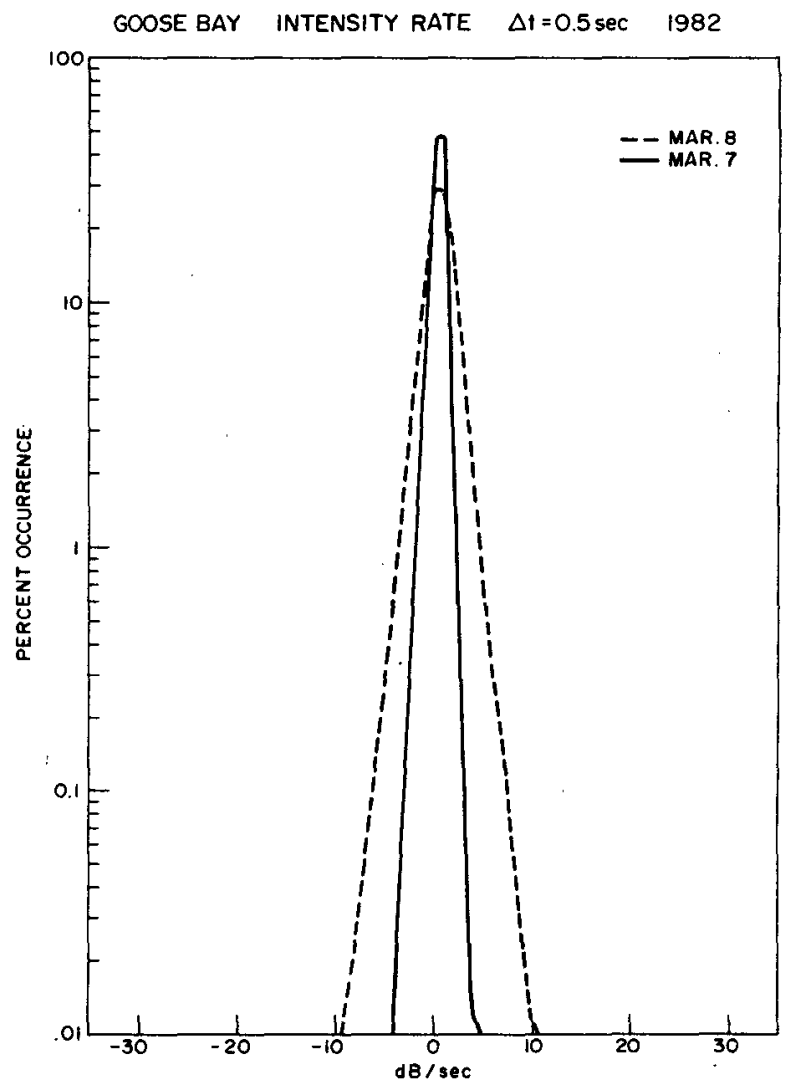

Fig. 13. The distribution of intensity rate of scintillations at $250 \mathrm{MHz}$ at Goose Bay on two successive nights with different ionospheric drifts. The drift was lower on Mar. 7 by a factor of 6 as compared to Mar. 8 .

speed is reflected in increased intensity rates. Fig. 14 shows the phase rate plots at the same frequency for the two days. It may be noted that at 0.1 percent level, the phase rate may attain values as high as $250^{\circ} \mathrm{s}^{-1}$ at an 


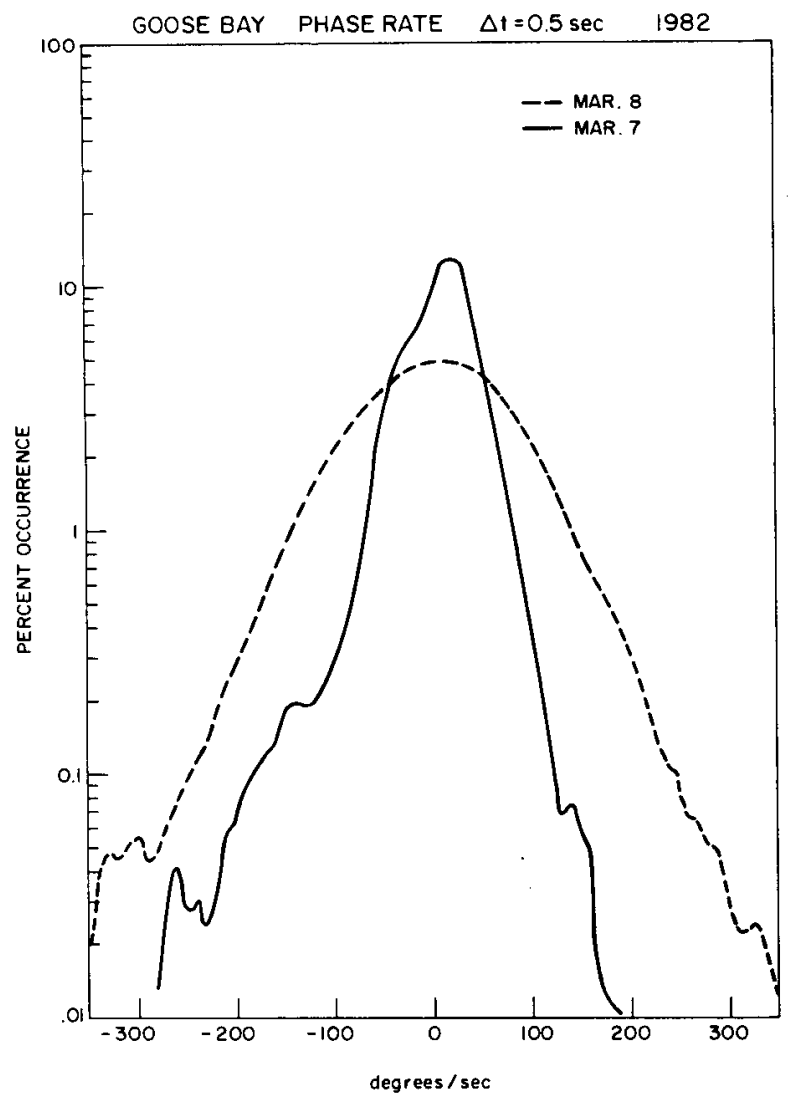

Fig. 14. Same as for Fig. 13, except here the distribution of phase rate of scintillations at $250 \mathrm{MHz}$ is shown.

auroral location when drift speed is high. If the change can be linearly extrapolated to smaller time intervals, the above value translates to $0.25^{\circ} \mathrm{ms}^{-1}$. This is an order of magnitude smaller than the phase rate encountered at Ascension Island and illustrated in Fig. 8(b).

\section{Summary}

The results on the structure of multifrequency amplitude scintillations covering the $250 \mathrm{MHz}-4 \mathrm{GHz}$ band of frequencies at the crest of the equatorial anomaly and the structure of approximately $250 \mathrm{MHz}$ phase and amplitude scintillations in the equatorial anomaly, auroral, and polar cap regions can be summarized as follows.

At Ascension Island, located at the crest of the equatorial anomaly, the most disturbed irregularity environment on a global basis was encountered during the last sunspot maximum period. At this location, the disturbance level was so intense that not only the $250 \mathrm{MHz}$ but also the $1541 \mathrm{MHz}$ transmissions from geostationary satellites exhibited saturated intensity scintillations which covered the $30 \mathrm{~dB}$ dynamic range of receivers that were used to record the satellite transmissions. Even the transmissions at $4 \mathrm{GHz}$ registered fluctuations as large as $6 \mathrm{~dB}$. The median and the 90th percentile values of rms phase deviation at $244 \mathrm{MHz}$ with $100 \mathrm{~s}$ detrend are 6 and 16 $\mathrm{rad}$, respectively, during the early evening hours. The phase spectral strengths (phase psd at $1 \mathrm{~Hz}$ ) which indicate the strength of turbulence are found to attain values as high as $-2 \mathrm{~dB}$. This is at least $10 \mathrm{~dB}$ above the most intense levels that are obtained at the polar cap or auroral stations. The extreme levels of turbulence at Ascension Island induce strong scattering of 257 and $1541 \mathrm{MHz}$ signals and thereby dictate the structures of amplitude and phase scintillations at both frequencies. The amplitude scintillation spectra at these frequencies under such situations show uniform psd up to fluctuation frequencies as high as a few hertz, and give rise to steep power law spectral indexes of -5 to -7 . The intensity decorrelation times are consequently reduced to values as low as 0.06 $\mathrm{s}$ at $257 \mathrm{MHz}$. The phase spectral slopes at $244 \mathrm{MHz}$ approach a value of -2.5 which is much shallower than is expected from either the in situ irregularity spectra or weak amplitude scintillations spectra at $4 \mathrm{GHz}$. This probably arises from sharp discontinuous phase changes or "cycle slips" [28] that develop due to phase diffraction effects under strong scatter conditions. In view of these discontinuous phase changes, extreme phase rates of 2 $3^{\circ} \mathrm{ms}^{-1}$ were observed. This is rather significant for UHF radar applications.

In the polar cap (Thule), the median and 90th percentile values of rms phase deviation at $250 \mathrm{MHz}$ for $82-\mathrm{s}$ detrend are observed to be 3 and $12 \mathrm{rad}$, respectively. The corresponding values for the $S_{4}$ index of scintillations are 0.5 and 0.8 . These values are in reasonable agreement with those published in [5] for another period of observations. The point of interest is the absence of any diurnal variation of polar cap scintillations, its annual variation with a pronounced minimum during local summer, and rather abrupt decrease of scintillations when the solar activity decreases below a threshold level. The irregularity spectral strengths in the polar cap or auroral stations are shown to be not as intense as in the equatorial region. At high latitudes, the phase spectral indexes of about -2.2 cannot be attributed to strong scattering effects, but are probably a result of shallow irregularity spectral indexes in the ionosphere. At auroral locations, the variability of ionospheric motion greatly controls the temporal structure of scintillations. The phase rates at $250 \mathrm{MHz}$ are typically of the order of $0.2-0.3^{\circ} \mathrm{ms}^{-1}$, but may show considerable changes due to the variability of ionospheric motion.

\section{ACKNOWLEDGMENT}

These observations have been made possible by the cooperation of many individuals and organizations. M.D. Cousins of SRI International designed the phase recording system. The initial software development was done by R.C. Livingston of SRI International. The efforts of F. Roberts of Logicon in modifying and adapting the software to the AFGL system are greatly appreciated. The authors would like to thank the Canadian Marconi Company and the Danish Arctic Contractors for their assistance in obtaining the Goose Bay and Thule data, respectively. 


\section{REFERENCES}

[1] J. Aarons, "Global morphology of ionospheric scintillation," Proc. IEEE, vol. 70 , pp. $360-378,1982$.

[2] R. C. Livingston, "Comparison of multifrequency equatorial scintillation: American and Pacific sectors," Radio Sci., vol. 15, p. '801, 1980.

[3] C. L. Rino and S. J. Matthews, "On the morphology of auroral zone radio wave scintillation," J. Geophys. Res., vol. 85 , p. $4139,1980$.

[4] S. Basu, S. Basu, R. C. Livingston, H. E. Whitney, and E. MacKenzie, "Comparison of ionospheric scintillation statistics from the North Atlantic and Alaskan sectors of the auroral oval using the WIDEBAND satellite," Air Force Geophys. Lab., Hanscom AFB, MA, Rep. AFGL-TR-81-0266, AD A111871, Sept. 1981.

[5] S. Basu, S. Basu, E. MacKenzie, and H. E. Whitney, "Morphology of phase and intensity scintillations in the auroral oval and polar cap," Radio Sci., vol. 20, pp. 347-356, 1985.

[6] C. L. Rino, R. C. Livingston, R. T. Tsunoda, R. M. Robinson, J. F. Vickrey, C. Senior, M. D. Cousins, J. Owen, and J. A. Klobuchar, "Recent studies of the structure and morphology of auroral zone F-region irregularities," Radio Sci., vol. 18, pp. 1167-1180, 1983.

[7] S. Basu and S. Basu, "Equatorial scintillations: Advances since ISEA6," J. Atmos. Terr. Phys., vol. 47, pp. 753-768, 1985.

[8] S. Basu, S. Basu, C. Senior, D. Weimer, E. Nielsen, and P. F. Fougere, "Velocity shears and sub-km scale irregularities in the nighttime auroral F-region,"' Geophys. Res. Lett., vol. 13, p. 101, 1986.

[9] E. J. Fremouw and J. A. Secan, "Modeling and scientific application of scintillation results," Radio Sci., vol. 19, pp. 687-694, 1984.

[10] S. Basu, S. Basu, R. C. Livingston, E. MacKenzie, and H. E. Whitney, "Phase and amplitude scintillation statistics at $244 \mathrm{HMz}$ from Goose Bay using a geostationary satellite," Air Force Geophys. Lab., Hanscom AFB, MA, Rep. AFGL-TR-82-0222, AD A124291, Aug. 1982.

[11] S. Basu, E. MacKenzie, S. Basu, H. C. Carlson, D. A. Hardy, F. J. Rich, and R. C. Livingston, "Coordinated measurements of low-energy electron precipitation and scintillations/TEC in the auroral oval," Radio Sci., vol. 18, p. 1151, 1983.

[12] H. E. Whitney and S. Basu, "The effect of ionospheric scintillation on VHF/UHF satellite communications," Radio Sci., vol. 12, p. 123, 1977.

[13] J. Aarons, H. E. Whitney, E. MacKenzie, and S. Basu, "Microwave equatorial scintillation intensity during solar maximum," Radio Sci., vol. 16 , pp. 939-945, 1981

[14] J. P. Mullen, E. MacKenzie, S. Basu, and H. E. Whitney, "UHF/ $\mathrm{GHz}$ scintillation observed at Ascension Island from 1980 through 1982,"'Radio Sci., vol. 20, pp. 357-365, 1985.

[15] B. H. Briggs and I. A. Parkin, "On the variation of radio star and satellite scintillation with zenith angle," J. Atmos. Terr. Phys., vol. 25, pp. 339-365, 1963.

[16] C. L. Rufenach, "Wavelength dependence of radio scintillation: Ionosphere and interplanetary irregularities," J. Geophys. Res., vol. 79 , pp. $1562-1566,1974$

[17] H. E. Whitney, "Notes on the relationship of scintillation index to probability distributions and their uses for system design," Air Force Cambridge Res. Lab., Rep. AFCRL-TR-74-0004, AD778092, Bedford, MA, 1974.

[18] S. Basu, S. Basu, J. P. McClure, W. B. Hanson, and H. E. Whitney, "High-resolution topside in-situ data of electron densities and VHF/ GHz scintillations in the equatorial region," J. Geophys. Res., vol. 88 , p. $403,1983$.

[19] S. J. Franke and C. H. Liu, "Observations and modeling of multifrequency VHF and $\mathrm{GHz}$ scintillations in the equatorial region," $J$. Geophys. Res, , vol. 88, p. 7075, 1983.

[20] S. Basu and H. E. Whitney, "The temporal structure of intensity scintillations near the magnetic equator," Radio Sci., vol. 18, p. 263, 1983.

[21] C. L. Rino, "Numerical computations for a one-dimensional power law phase screen," Radio Sci., vol. 15, p. 41, 1980.

[22] H. G. Booker and G. MajidiAhi, "Theory of refractive scattering in scintillation phenomena," J. Atmos. Terr. Phys., vol. 43, p. 1199, 1981.

[23] R. Umeki, C. H. Liu, and K. C. Yeh, "Multifrequency studies of ionospheric scintillations," Radio Sci., vol. 12, p. 311, 1977.

[24] M. Nakagami, "The $m$-distribution-A general formula of intensity distribution of rapid fading," in Statistical Methods of Radio Wave Propagation, W. C. Hoffman, Ed. Elmsford, NY: Pergamon, Symposium Publications Div., 1980.
[25] H. E. Whitney, J. Aarons, R. S. Allen, and D. R. Seeman, "Estimation of the cumulative amplitude probability distribution function of ionospheric scintillations," Radio Sci., vol. 7, pp. 1095-1104, 1972.

[26] C. L. Rino and J. Owen, "The time structure of transionospheric radio wave scintillation," Radio Sci., vol. 15, p. 479, 1980.

[27] P. F. Fougere, "On the accuracy of spectrum analysis of red noise processes using maximum entropy and periodogram methods: Simulation studies and application to geophysical data,"J. Geophys. Res., vol. 90 , pp. $4355-4366,1985$.

[28] C. L. Rino and J. Owen, "Numerical simulations of intensity scintillation using the power law phase screen model," Radio Sci., vol. 19 , p. 891,1984

[29] E. J. Fremouw, R. L. Leadabrand, R. C. Livingston, M. D. Cousins, C. L. Rino, B. C. Fair, and R. A. Long, "Early results from the DNA Wideband satellite experiment-Complex-signal scintillation," Radio Sci., vol. 13, p. 167, 1978.

[30] J. A. Whalen, " Auroral oval plotter and nomograph for determining corrected geomagnetic local time, latitude and longitude for high latitudes in the northern hemisphere,"' Air Force Cambridge Research Laboratory, Bedford, MA, Rep. AFCRL-TR-70-0422, AD 713170, 1970.

[31] J. Aarons, J. P. Mullen, H. E. Whitney, A. Johnson, and E. Weber, "VHF scintillation activity over polar latitudes,"' Geophys. Res. Lett., vol. 8, p. 277, 1981.

[32] J. F. Vickrey and M. C. Kelley, "The effects of a conducting E layer on classical F-region cross-field plasma diffusion,"'J. Geophys. Res., vol. 87, p. $4461,1982$.

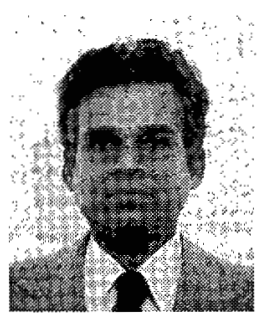

Santimay Basu received the Ph.D. degree in radio physics and electronics in 1964 from Calcutta University, India.

He was a Reader at the Institute of Radio Physics and Electronics from 1965 to 1975 with a sabbatical break in 1971 and 1972 when he was a Senior National Academy of Sciences Resident Research Associate at the Air Force Geophysics Laboratory, Hanscom AFB, MA. His teaching responsibilities at Calcutta included microwave transmission systems, antennas, and ionospheric radio propagation. He built up an active research group at Calcutta University specializing in low latitude radio propagation problems. In 1976 he joined Emmanuel College, Boston, MA, as a Physicist where he was instrumental in organizing intensive studies of ionospheric irregularities and their impact on communications systems. He joined the Ionospheric Effects Branch of AFGL in 1985 where he is responsible for organizing and conducting research on ionospheric structuring, their effects on radio wave propagation, and on ionospheric instability processes induced by high power radio waves. He has published more than 50 journal articles in the areas of natural and artificial irregularities and communication problems at high and low latitudes.

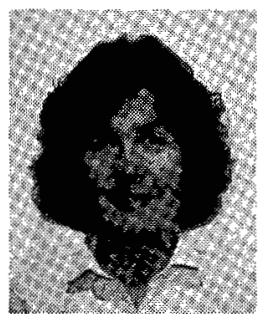

Eileen Martin MacKenzie was born in Boston, MA, in 1951. She received the M.S. degree in .physics from Northeastern University, Boston, in 1978.

She is a Physicist in the Physics Research Division of Emmanuel College, Boston. Since 1972 she has been involved in developing large scintillation databases, statistical analyses, and modeling efforts. Additional work has included coordination of scintillation parameters with in situ data, such as auroral imagery and particle precipitation patterns. 


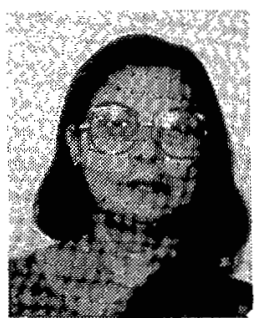

Sunanda Basu received the Ph.D. degree in 1972 in radio physics from Calcutta University, India.

She continued as a Research Associate at Calcutta University until 1975. In 1975 she accepted a National Academy of Sciences Resident Research Associateship at the Air Force Geophysics Laboratory, Hanscom AFB, MA, and since 1978 has been at Emmanuel College, Boston, MA, as a Physicist. Her major interest is in multitechnique studies of natural and artificial ionospheric irregularities; and she has organized many intensive campaigns for their study at the magnetic equator and at high latitudes with support from AFGL, NSF, and NASA.

Dr. Basu was a member of the Guest Investigator Team on the NASA Atmosphere Explorer Program and is currently a member of the Dynamics Explorer Guest Investigator Team. She was an Associate Editor of the Journal of Geophysical Research (Space Physics) during 1983-1985 and has recently been elected President of the Hanscom Chapter of Sigma Xi.

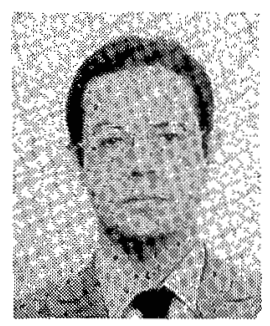

Emanoel Costa received the B.S. and M.S. degrees in electrical engineering from the Military Institute of Engineering (IME), Rio de Janeiro, Brazil, in 1972 and 1974 , respectively. He received the Ph.D. degree from Cornell University, Ithaca, NY, in 1977.

Since 1978 he has been with the Pontifical Catholic University of Rio de Janeiro (PUC/RJ), Brazil, where he is an Associate Professor at the Center for Telecommunication Studies (CETUC). On a sabbatical leave from this position, he spent the period between November 1984 and June 1986 at the Physics Research Division, Emmanuel College, Boston, MA, working on space-receiver analysis of ionospheric irregularity anisotropies and drifts. His research interests are in the areas of tropospheric and ionospheric effects on radiowave propagation and telecommunication systems.

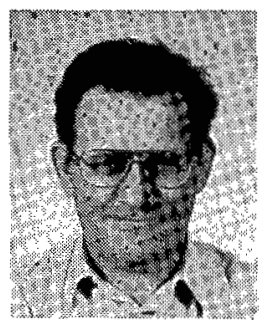

Paul F. Fougere (M'83-SM'85) received the B.S and M.S. degrees from Boston College, Boston, MA. In a part time effort he obtained the Ph.D. degree from Boston University with a dissertation on the electronic structure of diatomic molecules.

He spent a year at the Naval Research Laboratory in Washington, DC, working on ultrasonics, and another year at the General Electric Company in Lynn, MA. At this point he joined the Geomagnetism Branch at the Air Force Cambridge Research Center (now known as the Air
Force Geophysics Laboratory, AFGL). At AFGL he has worked on magnetic field analysis, especially spherical harmonic analysis; one of his models became part of the first International Geomagnetic Reference Field. Later work centered on theory and application of maximum entropy power spectral analysis, which he is currently applying, within the Ionospheric Effects Branch, to a large number of problems of interest to AFGL, such as ionospheric scintillation, ionospheric densities and velocities, neutral air densities, electric field data, Fourier transform spectroscopy, and Fourier transform mass spectrometry.

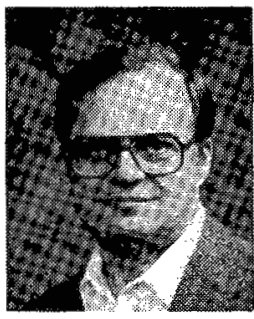

Herbert C. Carlson, Jr. was born in Brooklyn, $\mathrm{NY}$, in 1937. He received the Ph.D. degree in radio propagation from Comell University, Ithaca, $\mathrm{NY}$, in 1965.

In 1973, leaving the position of Head of the Ionospheric Physics Department at Arecibo, he went to the University of Texas at Dallas to participate in the beginning of the Atmospheric Explorer Satellite Program. He served at the National Science Foundation from 1977 to 1979 as Aeronomy Program Director, while also developing a major new National Science Foundation initiative in atmospheric sciences. From 1979 to 1981 he served as its first Program Manager for upper atmospheric facilities. He is Chief of the Ionospheric Effects Branch at the Air Force Geophysics Laboratory, Hanscom AFB, MA. His publications are primarily in the areas of ionospheric and plasma physics, ionospheric modification with high-power HF "heating," radar techniques applied to sensing ionospheric properties, and mesosphere-stratospheretroposphere winds, optical sensing of thermospheric properties, and highlatitude ionospheric irregularity processes as they affect RF systems.

Herbert E. Whitney, photograph and biography not available at the time of publication. 